\title{
Clinical prognostic factors in canine histiocytic sarcoma ${ }^{\dagger}$
}

\author{
N. G. Dervisis, M. Kiupel, Q. Qin and L. Cesario \\ VA-MD College of Veterinary Medicine, Blacksburg, VA, USA
}

\section{Keywords}

Bernese mountain dog, cancer, canine, CD18, corticosteroids, histiocytic sarcoma, sarcoma
Correspondence address: Nick Dervisis VA-MD College of Veterinary Medicine DSACS, Phase II, 205 Duck Pond Drive (0442)

Blacksburg, VA 24061, USA

e-mail:

dervisis@vt.edu

\begin{abstract}
Canine histiocytic sarcoma (HS) is an aggressive neoplasia with variable clinical course and fatal outcome. The goals of this study were to evaluate a large cohort of canine patients with immunohistochemically confirmed HS and identify clinical prognostic factors. Biopsy submissions to the Michigan State University with tentative HS diagnoses were histologically and immunohistochemically confirmed, medical records collected, and interviews with relevant veterinary clinics conducted. Of 1391 histopathology submissions with a diagnosis containing the word 'histiocytic', 335 were suspicious for malignancy, and 180 were consistent with HS and had adequate clinical information recorded. The most commonly represented breeds were Bernese mountain dogs $(n=53)$, labrador retrievers $(n=26)$ and golden retrievers $(n=17)$. Median survival for all dogs in the study was 170 days, and subgroup analysis identified palliative treatment, disseminated HS, and concurrent use of corticosteroids as statistically significant negative factors for survival, in both uni- and multi-variate methodologies.
\end{abstract}

\section{Introduction}

Canine histiocytic malignancies represent a group of neoplasms with shared morphological features, but diverse cellular origin and potentially divergent biologic behaviour. The malignant histiocytic disease complex was first recognized in Bernese mountain dogs and it is considered to be familial in this breed. ${ }^{1}$ Improved diagnostic techniques such as routine use of IHC and frozen section IHC allowing for accurate diagnosis, along with increased awareness for the disease, expanded the list of predisposed breeds to include golden retrievers, flat-coated retrievers, and rottweilers. ${ }^{2-4}$ The disease is characterized by proliferation and tissue infiltration of pleiomorphic histiocytic cells in multiple tissues. ${ }^{5,6}$ Neoplastic histiocytic infiltrates are most often found in the skin and subcutis, periarticular and articular tissues, spleen, liver, lung, bone marrow and lymph nodes, while less common

${ }^{\dagger}$ The work was carried out at the College of Veterinary Medicine Michigan State University. locations include ocular and central nervous system sites. $^{7-9}$

Histiocytic sarcoma (HS) may present as a localized tumour or disseminated disease, but eventually widespread metastasis occurs with a frequently fatal outcome. Three forms of the disease have been recognized based on the cellular origin of the tumour and the clinical manifestation of the disease: disseminated HS (DHS), localized HS (LHS) and hemophagocytic HS (HHS). ${ }^{10-14}$ The HHS is immunophenotypically distinct and carries a grave prognosis. ${ }^{13}$ Both localized and disseminated cases of canine HS arise from interstitial dendritic antigen presenting cells (DAPCs), but exhibit variable clinical behaviour. ${ }^{3,8,10,14-18}$ Localized and disseminated forms of HS present with identical histopathological features and manifest as poorly demarcated masses composed of pleomorphic, large, individualized round cells, or densely packed bundles of plump spindle cells. ${ }^{8}$ The neoplastic cells of HS express CD1, CD11c, Cd18, MHC II, ICAM-1, CD80, 
CD86, CD90, consistent with an interstitial DAPC phenotype.

Despite reported metastatic rates of $70-91 \%$, treatment for dogs with HS consisting of surgery, radiation, chemotherapy or a combination, appears to improve survival outcome. ${ }^{3,4,14,19}$ In a study of 59 dogs with DHS, $54 \%$ of dogs that lived longer than 7 days following the first treatment with CCNU responded to therapy, and responders had a median survival time of 5.5 months. ${ }^{10}$ LHS manifests as a primary tumour that most commonly develops in the skin and subcutis, although it may also be found in periarticular tissues surrounding large appendicular joints, or in the spleen, lymph nodes, lung or bone marrow. ${ }^{8,10,11,14}$ Dogs diagnosed with LHS appear to have a better prognosis when treated compared to dogs diagnosed with DHS, having median survival times of up to 18.9 months. ${ }^{14,15}$

The goal of this retrospective study was to analyse a large cohort of patients with histologically and immunohistochemically confirmed HS, in order to identify clinical prognostic factors, breed incidence, and to better predict survival times for animals treated for the localized and disseminated forms of the disease.

\section{Materials and methods}

\section{Study population}

The Michigan State University Diagnostic Center for Population and Animal Health (DCPAH) database was queried for surgical biopsy and necropsy submissions with a diagnosis including the word 'histiocytic', from January 2006 through December 2011. Reports with a diagnosis suggestive of neoplasia were selected for further analysis, while patients with a diagnosis suggestive of inflammatory, degenerative or other non-neoplastic disease process were excluded. Phone calls were placed to all veterinary clinics which had submitted specimens of interest. Permission to retrieve the clinical information for each specimen was granted and medical record copies were used for analysis. The following information was obtained from these records: patient signalment, tumour location, clinical signs and duration of signs, staging tests performed, method of diagnosis, treatment and evidence of response to treatment, disease progression, previous orthopaedic/surgical intervention, cause and date of death (if dead) and necropsy findings (if performed).

\section{Establishing diagnosis}

All tissues sample submissions suggestive of a neoplastic disease were reviewed by a single Board Certified pathologist (M. K.). Samples consistent with HS were submitted for immunohistochemistry (IHC) against CD18. Tumour samples for which CD18 was negative were excluded from the study. CD11d IHC was performed on all specimens for which clinical features were suspicious for HHS (splenic tumour location, presence of thrombocytopenia, anaemia and evident erythrophagocytosis on histopathology). Additional immunohistochemistry for CD3, CD20, Mum-1, tryptase and Thy-1 was performed as needed according to the microscopic features. All IHC stained slides, were reviewed by a single pathologist (M. K.).

\section{Staging}

Patients were considered fully staged if the following evaluation of both the thorax and abdomen was performed: three-view thoracic radiographs with any of the following tests: abdominal ultrasound, abdominal ultrasound and abdominal radiographs, abdominal computed tomography (CT) scan, abdominal exploratory surgery. Patients were considered partially staged if the following evaluation of the thorax and/or abdomen was performed: three-view thoracic radiographs with abdominal radiographs, three-view thoracic radiographs without abdominal imaging, abdominal ultrasound without thoracic radiographs, abdominal radiographs without thoracic radiographs, or abdominal exploratory surgery without thoracic radiographs. Patients without thoracic or abdominal imaging were considered not staged.

\section{Statistical analysis}

Overall survival (OS) was defined as the time from diagnosis until patient death due to the disease (HS). Patient death due to the disease was assumed to be due to HS, unless the Medical Records 
indicated otherwise. Patients were censored if they were still alive at the time of analysis, died due to other causes (necropsy confirmation), or lost to follow-up. All necropsy diagnosed dogs were excluded from the survival analysis, because they were euthanized before a diagnosis was established.

'Palliative treatment' was defined as any symptomatic treatment in the absence of treatment with chemotherapy, surgery, and/or radiation. 'Definitive treatment' was defined as treatment with surgery, chemotherapy, radiation, or any combination of these modalities. For statistical purposes, retriever breeds (golden retrievers, labrador retrievers, and flat-coated retrievers) were analysed as a single group termed 'retrievers'. Cases were classified as DHS if multiple tumour lesions were identifiable in more than one tissue based on physical examination and imaging data. Cases were classified as LHS if a single primary tumour +/regional lymph node metastasis were identified based on physical examination and imaging data. In cases of ambiguity, the case was classified as DHS. Cases with absence of staging information based on physical examination and imaging data were classified as undetermined. DHS cases stating positive for CD11d were classified as HHS.

The Kaplan-Meier survival analysis method was used to estimate OS curves following treatment. The Log-Rank test was used to compare the effect of potential risk factors (breed, sex, neuter status, age, body weight, presence of lymph node metastasis, presence of distant metastasis, localized versus disseminated disease, palliative treatment, surgery, chemotherapy, corticosteroids). The Cox proportional hazards regression method was used to determine whether potential risk factors (breed, sex, neuter status, age, body weight, presence of lymph node metastasis, presence of distant metastasis, localized versus disseminated disease, palliative treatment, surgery, chemotherapy, corticosteroids) were associated with OS following treatment. The potential risk factors were entered in the regression model if their $P<0.05$ and removed if $P>0.1$.

The chi-square test and chi-square test for trend were used to compare the incidence of DHS and LHS between different dog breeds, the D'Agostino-Pearson test was used to test for normality, and the Mann-Whitney test for independent samples was used to test for differences between non-normally distributed data.

All reported $P$-values were two-sided and $P$-values $<0.05$ were considered statistically significant. Statistical analyses were performed with standard software (MedCalc Statistical Software version 15.8 (MedCalc Software bvba, Ostend, Belgium; https://www.medcalc.org; 2015).

\section{Results}

\section{Database query results}

The search of the DCPAH database identified 1391 surgical biopsy and necropsy submissions with a diagnosis containing the word 'histiocytic'. Of these, 335 submissions (24\%) were suspicious for histiocytic sarcoma based on histopathological features. Positive IHC against CD18 was initially demonstrated for 102 (30.4\%) cases. IHC for CD18 was performed on all remaining tumour samples for which paraffin blocks were available $(N=213)$. Of these 213 samples, 108 had positive labelling for $\mathrm{CD} 18$, and 85 were negative for CD18. A total number of $210(62.7 \%)$ cases were diagnosed as HS based on morphologic and immunohistochemical features. Finally, 180 of these cases had medical records available and underwent further analysis.

\section{Patient characteristics}

Thirty-six distinct breeds were represented in the dataset. The most commonly represented breeds were Bernese mountain dogs $(n=53)$, mixed breed dogs $(n=28)$, labrador retrievers $(n=26)$, golden retrievers $(n=17)$, rottweilers $(n=8)$, schnauzers $(n=4)$, Shetland sheepdogs $(n=4)$, flat-coated retrievers $(n=3)$ and beagles $(n=3)$. The remaining dogs $(n=34)$ represented 28 different breeds. There were 88 male and 92 female dogs diagnosed with HS; $25 \%$ of males $(n=22)$ and $11.9 \%$ of females $(n=11)$ were intact.

In 178 of 180 cases, the age of the patient was known. The mean age at the time of diagnosis was 10.2 years $( \pm 3.0$; range $1.7-19)$. Bernese mountain dogs were diagnosed with HS at an earlier age when compared to retrievers (9.2 versus 10.4 years, $P=0.0236$; chi-square for trend $=5.527$, $P=0.0187)$. 
Table 1. Presenting clinical signs. 'Mass' includes cutaneous and subcutaneous location or 1 or multiple masses. 'Other' includes 1 of each: hematuria, lymphadenopathy, distended abdomen, increased lung sounds, seizure, regurgitation, polydipsia/polyuria, gastrointestinal signs not otherwise specified, and incidental. For 19 dogs, no presenting clinical signs were noted in their medical records

\begin{tabular}{ll}
\hline Clinical sign & \# (\%) \\
\hline Mass & $70(43.2 \%)$ \\
Lethargy & $28(17.3 \%)$ \\
Lameness & $19(11.7 \%)$ \\
Anorexia & $13(8.0 \%)$ \\
Weight Loss & $12(7.4 \%)$ \\
Swelling & $4(2.5 \%)$ \\
Dyspnea & $3(1.9 \%)$ \\
Effusion & $2(1.2 \%)$ \\
Collapse & $2(1.2 \%)$ \\
Other & $9(5.6 \%)$ \\
\hline
\end{tabular}

\section{Disease characteristics}

Presenting clinical signs were available for 168 dogs. In 1 dog, the HS diagnosis was incidental. The most common presenting clinical signs were presence of a mass (43.2\%), lethargy (17.3\%), and lameness (11.7\%), with the other clinical signs spanning a large spectrum (Table 1). The localization of $\mathrm{HS}$ on presentation was variable. The organs most commonly involved were the skin or subcutaneous tissues, spleen, lymph nodes, lungs or liver, together representing $73.5 \%$ of the cases. Bone and/or joint localization accounted for $18.3 \%$ of the cases, including elbow/axilla, stifle, shoulder, humerus, femur, hock and temporomandibular joint (Table 2).

The duration of clinical signs prior to diagnosis was recorded for 160 cases. The overall median duration was 24.5 days (range: $0-758$ ). Dogs diagnosed with LHS had a significantly longer median duration of clinical signs prior to diagnosis (42.5 days) when compared to dogs with DHS (17.5 days) $(P=0.0279)$.

Forty-three dogs (24\%) were fully staged at the time of diagnosis. These patients had three-view thoracic radiographs and either abdominal ultrasound $(n=39)$, abdominal ultrasound and abdominal radiographs $(n=2)$, abdominal CT scan $(n=1)$ or abdominal exploratory surgery $(n=1)$. Seventy-two patients (40\%) underwent partial staging evaluation: three-view thoracic radiographs
Table 2. Anatomic lesion location at the time of presentation. "Bone/Joint" includes elbow/axilla, stifle, shoulder, humerus, femur, hock, and temporomandibular joint. "Mass" includes cutaneous and subcutaneous location of one or multiple masses. "Other" includes one of each: gall bladder, bone marrow, tarsus, carpus, spine, tibia, pelvis, antebrachium, eyelid.

\begin{tabular}{lc}
\hline Sites affected & $\#(\%)$ \\
\hline Mass & $49(18.6 \%)$ \\
Bone/joint & $48(18.3 \%)$ \\
Spleen & $43(16.4 \%)$ \\
Lymph node & $43(16.4 \%)$ \\
Lung & $30(11.4 \%)$ \\
Liver & $28(10.7 \%)$ \\
Oral & $4(1.5 \%)$ \\
Kidney & $4(1.5 \%)$ \\
Digit & $3(1.1 \%)$ \\
Gastrointestinal track & $2(0.8 \%)$ \\
Other & $9(3.3 \%)$ \\
\hline
\end{tabular}

with abdominal radiographs $(n=8)$, three-view thoracic radiographs without abdominal imaging $(n=45)$, abdominal ultrasound without thoracic radiographs $(n=4)$, abdominal radiographs without thoracic radiographs $(n=10)$ and abdominal exploratory surgery without thoracic radiographs $(n=5)$. In 65 patients $(36 \%)$ neither thoracic nor abdominal organ imaging studies were performed.

Categorization of localized or disseminated HS could be determined for $99 / 180$ cases (55\%), while not sufficient staging data precluded categorization of $81 / 180$ (45\%) cases. Of the 52 dogs (52.5\%) diagnosed with LHS, 31 dogs (59.6\%) had the periarticular form of the disease (PAHS), while the rest were localized to the skin, subcutaneous tissues, lung and spleen. Of the 47 dogs (47.5\%) initially diagnosed with DHS, 6 cases (12.8\%) were consistent with the hemophagocytic form of the disease (HHS). There were 22 Bernese mountain dogs and 6 retrievers diagnosed with DHS and 13 Bernese mountain dogs and 21 retrievers diagnosed with LHS (Chi-squared $=8.588, P=0.0034$ ).

In 57 patients $(31.7 \%)$, lymphadenopathy was noted on physical examination, radiographs or ultrasonographic exam. In the remaining 123 cases, lymphadenopathy was either absent, not evaluated, or not reported. In 49 patients the lymphadenopathy was further evaluated with cytology or histopathology. Evidence of metastatic disease was confirmed in 43 of the $49(87.8 \%)$ cases, based 
Table 3. Definitive treatments administered of dogs diagnosed with the Disseminated (DHS), Hemophagocytic (HHS), Localized (LHS) forms of the disease. Undetermined refers to dogs that could not be classified to a specific form of HS due to lack of medical record data.

\begin{tabular}{lccccc}
\hline & DHS & HHS & LHS & Undetermined & Total \\
\hline Chemotherapy single modality & 10 & 2 & 0 & 4 & 16 \\
Surgery single modality & 7 & 0 & 17 & 44 & 68 \\
Radiation single modality & 0 & 0 & 2 & 0 & 2 \\
Chemotherapy and surgery & 4 & 0 & 15 & 4 & 23 \\
Chemotherapy and radiation & 0 & 0 & 6 & 0 & 6 \\
\hline
\end{tabular}

on cytologic $(n=22)$ or histopathologic $(n=21)$ evaluation. In six of the 49 cases, there was no evidence of metastatic disease.

\section{Treatments}

Detailed treatment information was available for 171 dogs. Sixty dogs (35\%) received palliative treatment, and 111 (65\%) dogs received definitive treatment. Palliative treatment included the use of corticosteroids $(n=13)$, opioids $(n=12)$, non-steroid anti-inflammatory drugs $(n=10)$, antibiotics $(n=8)$, antacids $(n=3)$, gabapentin $(n=2)$, antiemetics $(n=2)$, bisphosphonates (alendronate $n=1$, pamidronate $n=2)$ and holistic/ alternative medicine $(n=2)$.

Chemotherapy was administered in 45 dogs: 23 dogs were diagnosed with LHS (16 in adjuvant setting, and 7 at gross disease setting), 12 dogs diagnosed with DHS, 2 dogs diagnosed with HHS and in 8 dogs that were not fully staged. Chemotherapeutic agents included lomustine $(n=34)$, doxorubicin $(n=19)$, vincristine $(n=8)$, cyclophosphamide $(n=7)$, carboplatin $(n=4)$, melphalan $(n=2)$, temozolomide $(n=2)$, mitoxantrone $(n=1)$, bleomycin $(n=1)$, dacarbazine $(n=1)$, methotrexate $(n=1)$, cytosine arabinoside $(n=1)$, cladribine $(n=1)$, vinblastine $(n=1)$, toceranib $(n=1)$, chlorambucil $(n=1)$.

Surgery was performed in 91 dogs. In 34 cases the dogs were diagnosed with LHS, in 13 cases the dogs were diagnosed with DHS, and in 44 cases, the dogs were not fully staged prior to surgery. Of the 68 dogs that underwent surgery as the sole treatment modality, 17 had LHS, 7 had DHS and 44 dogs were not fully staged. Chemotherapy was administered in combination with surgery in 23 dogs; 15 dogs were diagnosed with LHS, 4 with DHS, and 4 were not fully staged.

Eight dogs received radiation therapy with either a coarse fractionation scheme (8Gy once weekly for 3 fractions, $n=4$ ) or a standard fractionated/ curative intent protocol (3Gy once daily for 18 fractions, $n=4)$. All dogs undergoing curative intent radiation therapy also received chemotherapy, while only 2 of the 4 dogs receiving coarse fractionated radiation therapy were treated with chemotherapy (Table 3 ).

For 168 dogs, there was adequate medical record data regarding the use of corticosteroids. Forty-five dogs received corticosteroids and 123 dogs did not receive corticosteroids. Eleven dogs (24.5\%) received corticosteroids as part of their palliative treatment, and 34 dogs (75.5\%) received corticosteroids concurrently with definitive treatment. Of these 34 dogs, 10 dogs were diagnosed with LHS, 12 with DHS and 12 were not able to be assigned to either LHS or DHS.

\section{Patient outcomes}

Of the 180 patients, 41 patients were diagnosed on necropsy and 10 dogs died within 7 days following diagnosis. For three dogs, no reliable survival data could be obtained. Survival data were available for a total of 180 dogs, and their median survival time (OS) was 170 days (95\% CI: 87 - 264). The median survival time for the subgroup of dogs surviving more than 7 days post diagnosis $(N=126)$ was 180 days (95\% CI: 118-349) (Fig. 1). Factors positively influencing OS on univariate analysis included definitive treatment, LHS, lack of lymph node metastasis, and lack of use of corticosteroids. Definitive treatment was associated with prolonged survival (349 versus 39 days, $P<0.0001$ ) 


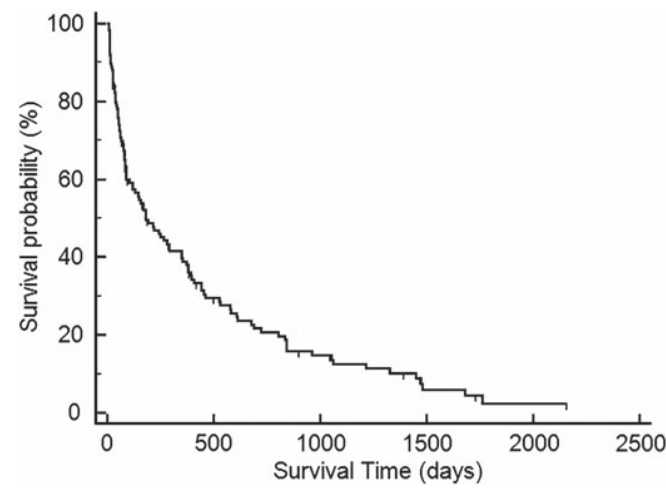

Figure 1. Kaplan-Meier survival curve estimating median OS (180 days) for dogs diagnosed with HS and surviving $>7$ days post diagnosis $(n=126)$. Vertical tick marks represent animals that were censored $(n=19)$.

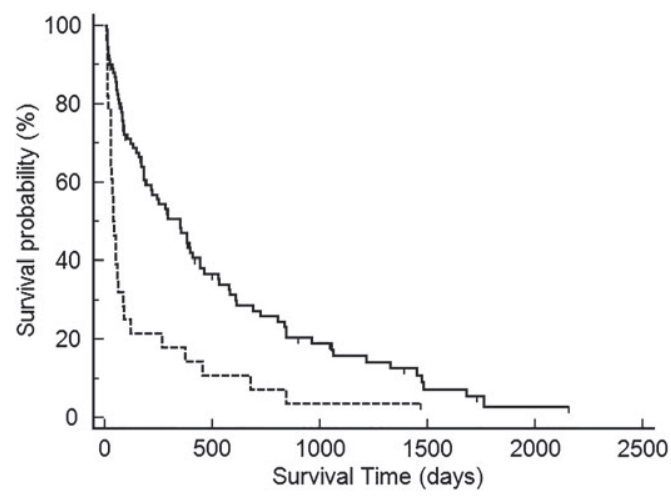

Figure 2. Kaplan-Meier survival curve estimating median OS for dogs diagnosed with HS receiving palliative treatment (dashed line; $n=28$ ) versus dogs with HS receiving definitive treatment (solid line; $n=93$ ). Dogs receiving definitive treatment had significantly $(P<0.0001)$ longer median OS (349 days) than dogs receiving palliative treatment (39 days). Vertical tick marks represent animals that were censored (palliative group $n=1$; definitive group $n=17)$.

(Fig. 2). Dogs diagnosed with LHS had median survival of 398 days compared to 78 days in dogs with DHS $(P=0.0002)$ (Fig. 3). Use of corticosteroids was associated with decreased survival (87 versus 349 days, $P=0.0081)$. In dogs diagnosed with LHS, the presence of lymph node metastasis was associated with decreased survival (180 versus 461 days, $P=0.0407$ ) (Fig. 4). Dog breed was not associated with differences in OS (Fig. 5). Two of the 6 dogs diagnosed with HHS were treated with chemotherapy, surviving for 50 and 129 days, respectively.

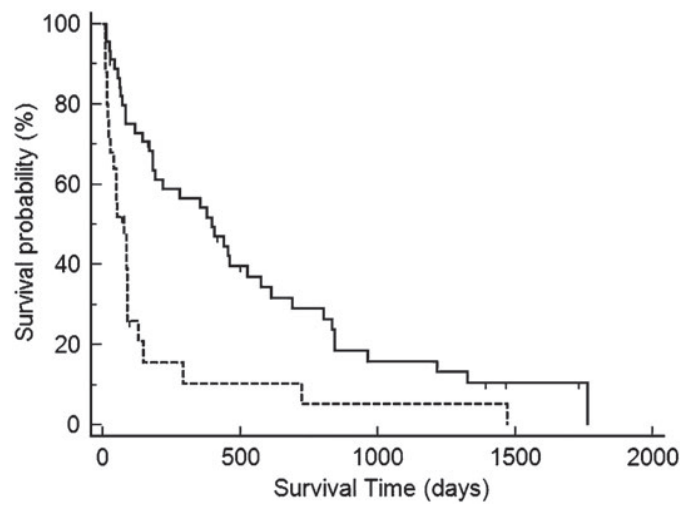

Figure 3. Kaplan-Meier survival curve estimating median OS for dogs diagnosed with LHS (solid line; $n=46$ ) compared to dogs with DHS (dashed line; $n=25$ ). Dogs diagnosed with LHS had significantly $(P=0.0002)$ longer median OS (398 days) than dogs diagnosed with DHS (78 days). Vertical tick marks represent animals that were censored (LHS group $n=8$; DHS group $n=2$ ).

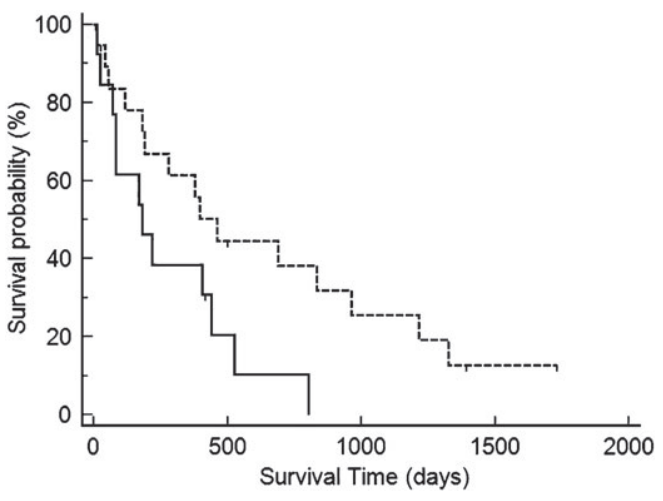

Figure 4. Kaplan-Meier survival curve estimating median OS for dogs diagnosed with LHS and evidence of lymph node metastasis (solid line; $n=13$ ) versus dogs with LHS without evidence of lymph node metastasis (dashed line; $n=20$ ). Dogs with lymph node metastasis had significantly $(P=0.0407)$ shorter median OS (180 days) than dogs without lymph node metastasis (461 days). Vertical tick marks represent animals that were censored (lymph node metastasis group $n=1$; dogs without lymph node metastasis $n=5)$.

For dogs receiving definitive treatment, factors associated with OS on univariate analysis were LHS and corticosteroid administration. Dogs diagnosed with LHS had median survival times of 406 days versus 85 days for dogs with DHS $(P=0.0097)$. Use of corticosteroids was associated with decreased survival (170 versus 394 days, $P=0.0456$ ) (Fig. 6). For dogs diagnosed with LHS that were treated with chemotherapy, use of surgery did not result 


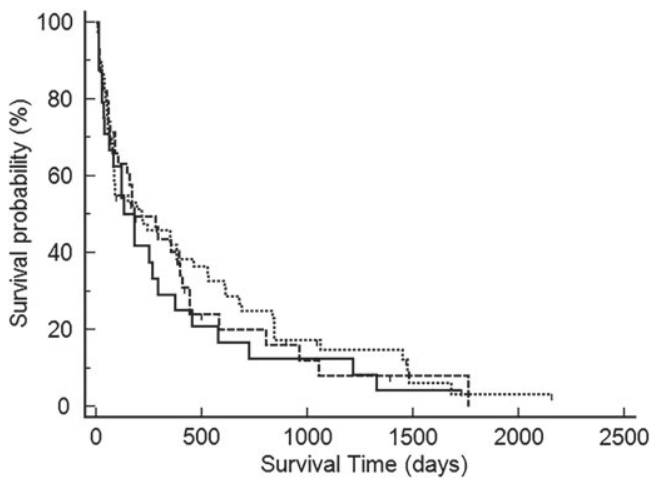

Figure 5. Kaplan-Meier survival curve estimating median OS for BMD dogs diagnosed with HS (solid line; $n=25$ ) versus retrievers (dashed line; $n=39$ ), versus all other breeds and mixed breed dogs (dotted line; $n=62$ ). No statistical difference $(P=0.63)$ was detected between BMDs (129 days), retrievers (180 days), and others (217 days). Vertical tick marks represent animals that were censored (BMD group $n=2$; retriever group $n=7$; others group $n=10$ ).

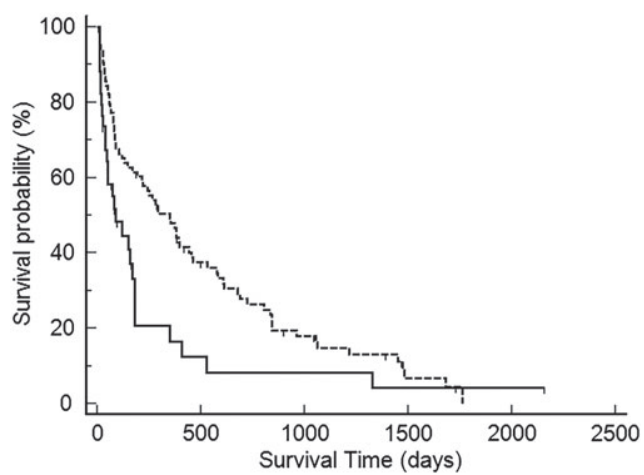

Figure 6. Kaplan-Meier survival curve estimating median OS for dogs receiving definitive treatment and concurrent corticosteroids (solid line; $n=28$ ) versus dogs receiving definitive treatment without concurrent corticosteroids (dashed line; $n=90)$. Dogs receiving corticosteroids had significantly $(P=0.0456)$ shorter median OS (170 days) than dogs not receiving corticosteroids (394 days). Vertical tick marks represent animals that were censored (corticosteroid group $n=6$; non-corticosteroid group $n=11$ ).

in a statistically significant difference in survival $(N=22, P=0.3077)$.

Factors associated with an increased risk of death that remained significant on multi-variate analysis were palliative treatment (relative risk, $R R=15.50$, $P=0.0001)$, DHS form of disease $(\mathrm{RR}=2.82$, $P=0.004)$ and concurrent use of corticosteroids $(\mathrm{RR}=2.14, P=0.0398)$.

Outcome was known for 158 dogs, while 22 dogs were lost to follow-up. One hundred and twenty-one dogs were euthanized, 18 died, and 19 were alive at the time of the analysis. No post mortem examination was reported for 128 cases (considered dead due to HS in our statistical analysis), necropsy at a pathology lab was performed in 22 cases, and partial necropsy or in-house necropsy in 8 cases. Dogs with partial or in-house necropsies were considered dead due to HS in our statistical analysis. Of the 22 cases with full necropsy results, 19 dogs were reported to have died due to HS, 1 dog died due to IMHA (censored in our statistical analysis), 1 dog due to post-surgical complications (pneumothorax, censored in our statistical analysis) and $1 \mathrm{dog}$ due to carcinomatosis 180 days post diagnosis (censored in our statistical analysis).

\section{Discussion}

Our study represents the largest, histopathologically and immunohistochemically confirmed clinical study of HS in the dog. We were able to identify three statistically significant negative prognostic factors consistently in uni- and multi-variate analysis: the disseminated form of the disease, palliative treatment, and concurrent use of corticosteroids. The dog breed was not associated with survival, although there was a statistically significant difference in the distribution of DHS and LHS between BMDs and retrievers. Our study further underscores the need for immunohistochemistry in order to establish a diagnosis consistent with HS in the dog. Of the 335 biopsy samples initially diagnosed as suspected HS neoplasia, only $62.7 \%$ had HS confirmed by IHC.

The disseminated form of the disease appears to be a consistent negative prognostic indicator in our dataset, confirming previously published data. ${ }^{10,15,16}$ In our study, dogs diagnosed with DHS had a median survival time of 85 days when treated beyond palliative care, compared to a median survival time of 406 days for dogs with LHS. In the multi-variate analysis, dogs with DHS were 2.82 times more likely to die when compared to dogs with LHS. This result may be attributed to the more advanced stage of the disease and the potentially more aggressive biological behaviour of DHS. Interpretation of these findings should take into account 
that some dogs assigned on the LHS group had lymph node metastasis, which can affect the appropriate classification of these dogs. However, despite the similar histomorphological appearance of DHS and LHS, the diseases exhibit divergent clinical behaviour in patients that undergo treatment.

Palliative treatment of HS has been associated with reduced survival. ${ }^{4,15}$ In our study, treatment with palliative care carried a grave prognosis for this group of dogs with a median survival time of 39 days. The true expected survival may be shorter than reported here, as our survival analysis excluded dogs that died or were euthanized within 7 days following diagnosis, and included dogs with LHS that may had a longer expected survival despite lack of definitive treatment. These results support treatment beyond palliative care for dogs diagnosed with HS.

Administration of corticosteroids has been previously reported as a negative prognostic factor for survival in a group of dogs diagnosed with PAHS. ${ }^{16}$ In our study, we analysed the effect of corticosteroid use in a relatively large group of dogs that received definitive treatment. Concurrent use of corticosteroids appears to increase the risk of death by 2.14 times compared to dogs with HS that did not receive corticosteroids along with other definitive treatment modalities. Despite the statistical significance of our results in the multi-variate analysis, it cannot be concluded whether corticosteroid administration is the cause of decreased survival or remains a confounding factor. Corticosteroid administration may have been associated with dogs in worse clinical condition, (confounding), inducing resistance to chemotherapeutics used, or may have led to increase of gastrointestinal side effects that would discouraged owners from continuing with chemotherapy. A prospective, blinded, randomized trial is necessary to confirm or reject this finding. Power analysis using our results, a 1:1 randomization ratio, with an alpha of 0.05 , and beta of 0.20 , indicates that a minimum of 62 dogs per group is required to detect a statistically significant survival difference at 6 months after the initial diagnosis.

The observed breed distribution in this study is consistent with the published literature. We were able to identify a small number of schnauzers $(n=4)$, a breed that has not previously been identified as affected. The incidence of DHS and LHS was significantly different between BMDs and retrievers, with BMDs more likely to be diagnosed with DHS. Additionally, BMDs were younger at the time of diagnosis when compared to the retrievers. Our findings may reflect the genetic background of the disease, as well the potential breed-specific genetic events responsible for the development of the disease. ${ }^{20}$ Despite this, dog breed was not a statistically significant factor associated with survival for dogs that received treatment beyond palliative therapy. The latter observation may indicate that while the genetic makeup of the breed has a role in the pathogenesis of HS, tumour behaviour seems independent of these initiating events.

This study has a number of weaknesses, the majority of which stem from the retrospective design. We were unable to control for confounding factors, analyse groups of dogs receiving standardized staging and therapy or confirm the cause of death for most patients. Hence, censoring was based on best available data, with many cases lacking full necropsy and thus the treatment-related deaths and/or IMHA case could have been incorrectly censored. Despite using IHC for CD18, we were unable to interrogate all tumours for the multitude of markers mentioned in the Introduction, because we had only access to paraffin embedded, formalin fixed tissues. Thus, it is possible that some dogs in our study may not have had HS, as currently defined in the literature. In order to reduce bias, the number of prognostic factors interrogated were reduced to only those clinical in nature, dogs were assigned to LHS or DHS groups only if there was adequate (full or partial) staging, and all histopathology and immunohistochemistry samples were reviewed by a single pathologist. Additionally, the survival analysis only included dogs that lived longer than 7 days following diagnosis to avoid bias introduced by patient data obtained at necropsy or for patients that were euthanized at the time of diagnosis. Dogs diagnosed with LHS appeared not to benefit from surgery in our analysis; this should be interpreted with extreme caution, as we had limited data available on the type of surgery these dogs had, which could severely affect the reported results. Finally, care should be given on 
the interpretation of our results, due to the relatively small numbers in some of our subgroup analyses. All conclusions from this study should ideally be confirmed with adequately controlled, prospective studies.

In conclusion, we were able to confirm previously reported clinical prognostic factors and identify a putative new one. Use of corticosteroids may have a negative effect in dogs diagnosed with HS, receiving definitive therapy. The need for an accurate diagnosis before treatment decisions are made cannot be overstated, especially when novel screening, diagnostic, or therapeutic options are offered.

\section{Acknowledgements}

We would like to thank Thomas Wood and Patty Schultz at DCPAH for their technical expertise in identifying and retrieving all paraffin blocks and performing immunohistochemistry. A special thanks to all of the referring veterinarians, technicians, staff and owners who generously donated time and effort to make this study possible.

This study was made possible with funding through the Department of Small Animal Medicine, College of Veterinary Medicine, Michigan State University, and Morris Animal Foundation (D08CA-308).

\section{References}

1. Moore PF. Systemic histiocytosis of Bernese mountain dogs. Veterinary Pathology 1984; 21: 554-563.

2. Dobson JM. Breed-predispositions to cancer in pedigree dogs. ISRN Veterinary Science 2013;2013:941275. 10.1155/2013/941275. 23738139 PMC3658424.

3. Craig LE, Julian ME, Ferracone JD. The diagnosis and prognosis of synovial tumors in dogs: 35 cases. Veterinary Pathology. 2002;39:66-73 12102220.

4. Rassnick KM, Moore AS, Russell DS, Northrup NC, Kristal O, Bailey DB, et al. Phase II, open-label trial of single-agent CCNU in dogs with previously untreated histiocytic sarcoma. Journal of Veterinary Internal Medicine. 2010;24:1528-1531 21155191.

5. Moore PF, Rosin A. Malignant histiocytosis of Bernese mountain dogs. Veterinary Pathology. 1986;23:1 - 103946051.

6. Rosin A, Moore P, Dubielzig R. Malignant histiocytosis in Bernese Mountain dogs. Journal of the American Veterinary Medical Association. 1986;188:1041 - 10453710888.

7. Naranjo C, Dubielzig RR, Friedrichs KR. Canine ocular histiocytic sarcoma. Veterinary Ophthalmology. 2007;10:179-185 10.1111/j.1463-5224.2007.00534.x. 17445080.

8. Affolter VK, Moore PF. Localized and disseminated histiocytic sarcoma of dendritic cell origin in dogs. Veterinary Pathology. 2002;39:74-83 12102221.

9. Chandra AM, Ginn PE. Primary malignant histiocytosis of the brain in a dog. Journal of Comparative Pathology. 1999;121:77-82 10.1053/jcpa.1998.029610373296.

10. Skorupski KA, Clifford CA, Paoloni MC, Lara-Garcia A, Barber L, Kent MS, et al. CCNU for the treatment of dogs with histiocytic sarcoma. Journal of Veterinary Internal Medicine. 2007;21:121-126 17338159.

11. Schultz RM, Puchalski SM, Kent M, Moore PF. Skeletal lesions of histiocytic sarcoma in nineteen dogs. Veterinary Radiology \& Ultrasound. 2007;48:539-543 18018725.

12. Fulmer AK, Mauldin GE. Canine histiocytic neoplasia: an overview. The Canadian Veterinary Journal. 2007;48:1041 - 1043,17987966 PMC1978291.

13. Moore PF, Affolter VK, Vernau W. Canine hemophagocytic histiocytic sarcoma: a proliferative disorder of CD11d+ macrophages. Veterinary Pathology. 2006;43:632-645 10.1354/vp.43-5-632 16966440

14. Fidel J, Schiller I, Hauser B, Jausi Y, Rohrer-Bley C, Roos M, et al. Histiocytic sarcomas in flat-coated retrievers: a summary of 37 cases (November 1998-March 2005). Veterinary and Comparative Oncology 2006; 4: 63-74.

15. Skorupski KA, Rodriguez CO, Krick EL, Clifford CA, Ward R, Kent MS. Long-term survival in dogs with localized histiocytic sarcoma treated with CCNU as an adjuvant to local therapy. Veterinary and Comparative Oncology. 2009;7:139- 144 10.1111/j.1476-5829.2009.00186.x 19453368.

16. Klahn SL, Kitchell BE, Dervisis NG. Evaluation and comparison of outcomes in dogs with periarticular and nonperiarticular histiocytic sarcoma. Journal of the American Veterinary Medical Association. 2011;239:90-96 10.2460/javma.239.1.90. 21718201.

17. van Kuijk L, van Ginkel K, de Vos JP, Brearley MJ, Butinar J, Gielen I, et al. Peri-articular histiocytic sarcoma and previous joint disease in Bernese mountain dogs. Journal of Veterinary Internal Medicine. 2013;27:293-299 10.1111 jvim. 1205923458888 . 
18. Mariani CL, Jennings MK, Olby NJ, Borst LB, Brown JC, Jr., Robertson ID, et al. Histiocytic sarcoma with central nervous system involvement in dogs: 19 cases (2006-2012). Journal of Veterinary Internal Medicine.

2015;29:607-613.10.1111/jvim.1255425711602.

19. Cannon C, Borgatti A, Henson M, Husbands B. Evaluation of a combination chemotherapy protocol including lomustine and doxorubicin in canine histiocytic sarcoma. The Journal of Small Animal
Practice. 2015;56:425-429 10.1111/jsap.12354 25828786.

20. Hedan B, Thomas R, Motsinger-Reif A, Abadie J, Andre C, Cullen J, et al. Molecular cytogenetic characterization of canine histiocytic sarcoma: A spontaneous model for human histiocytic cancer identifies deletion of tumor suppressor genes and highlights influence of genetic background on tumor behavior. BMC Cancer 2011;11:201 10.1186/1471-2407-11-201 21615919 PMC3121728. 